SHORT REPORT

\title{
Sexually transmitted infections in women who have sex with women
}

\author{
J V Bailey, C Farquhar, C Owen, P Mangtani
}

Sex Transm Infect 2004;80:244-246. doi: 10.1136/sti.2003.007641

Objectives: To describe the prevalence of sexually transmitted infection (STIs) in a sample of women who have sex with women (WSW) and to identify risk factors for the acquisition of STI.

Method: Cross sectional survey. Questionnaire for demographic, sexual history, and sexual practice data linked with the results of genitourinary examination. 708 new patients attending two sexual health clinics for lesbians and bisexual women in London were surveyed.

Results: A majority of WSW reported sexual histories with men (82\%). Bacterial vaginosis and candida species were commonly diagnosed $(31.4 \%$ and $18.4 \%$ respectively). Genital warts, genital herpes, and trichomoniasis were infrequently diagnosed $(1.6 \%, 1.1 \%$, and $1.3 \%$ respectively). Chlamydia, pelvic inflammatory disease, and gonorrhoea infections were rare $(0.6 \%, 0.3 \%$, and $0.3 \%$ respectively) and diagnosed only in women who had histories of sex with men. Conclusions: Although we have demonstrated a low prevalence of STI, WSW may have sexual histories with men as well as women or histories of injecting drug use, and are therefore vulnerable to sexually transmitted or blood borne infections. Diagnosis of trichomoniasis, genital herpes, and genital warts in three women who had no history of sex with men implies that sexual transmission between women is possible.

$\mathrm{T}$ here are no routinely collected data concerning transmis sion of sexually transmitted infections (STIs) between women in the United Kingdom. Data from genitourinary medicine (GUM) clinics analysed by the Public Health Laboratory Service Communicable Disease Surveillance Centre do not include female homosexual contact. ${ }^{1}$

Health carers may make the assumption that having a lesbian identity precludes sexual activity with men or that transmission of STI between women is unlikely. ${ }^{2}$ We present data describing the prevalence and risk factors for STI in a sample of 708 women who have sex with women (WSW).

\section{METHOD}

Ethical permission was obtained, and all new patients at two lesbian sexual health clinics in London were offered a questionnaire to gather data about potential risk factors for STI: demographic features, sexual history, and sexual practice. Between 1992 and 1995, 803 patients completed questionnaires out of an estimated 1000 attenders. No record of non-responders was kept. A total of 708 out of 803 had a GU examination and results from this were linked with questionnaire responses. This study includes 199 women in an initial prevalence study of STI in $\mathrm{WSW}^{3}$; we present here the results from the complete study population focusing on sexual history and other risk factors for STI.

A GU examination comprised cervical and urethral Gram stained smear and culture for Neisseria gonorrhoeae, cervical swab for Chlamydia trachomatis by ELISA, and high vaginal samples for Trichomonas vaginalis by wet preparation. Gram stained vaginal preparations were taken for Candida $s p$ identification and culture. Bacterial vaginosis (BV) was diagnosed using Amsel's criteria. Screening for treponemal disease used the Venereal Disease Research Laboratory (VDRL) test and the Treponema pallidum haemagglutination assay (TPHA). Herpes simplex cultures were performed as clinically indicated and genital warts were diagnosed by visual inspection. Pelvic inflammatory disease (PID) was diagnosed on clinical examination with a raised white cell count.

\section{Statistical analysis}

Diagnosis of one or more STIs (excluding candida and BV) in women who had had a GU screen was the outcome of interest. Variables reaching statistical significance at the level of $\mathrm{p}<0.1$ in univariate analysis were included in a multivariate model, as well as variables related to STI in other studies. A logistic regression model was built up in a stepwise fashion, using the likelihood ratio test and observing change in odds ratios.

\section{RESULTS}

Description of the sample $(n=708)$

The majority of the sample (92\%) identified as lesbian, $7 \%$ as bisexual; $65 \%$ were employed, $21 \%$ were unemployed, and $10 \%$ were students. Most $(90 \%)$ came into the category of white ethnicity, and the sample had a mean age of 31 years (SD 6.4). Fifty five per cent were asymptomatic.

\section{Prevalence of sexually transmitted infections}

There were no significant differences in prevalence of STIs when stratified by history of sex with men (table 1). However PID, chlamydia and gonorrhoea were diagnosed only in women with a history of sex with men.

Three women who had not been sexually active with men were diagnosed with one of the following: genital warts, primary genital herpes, and trichomoniasis. All three women reported engaging in a large variety of sexual practices with female partners so it is not possible to draw conclusions about modes of transmission.

Two women were diagnosed with hepatitis C; both had histories of injecting drug use. There were no cases of HIV, syphilis, or hepatitis B. However, while most subjects were tested for syphilis, a minority were tested for HIV or hepatitis, so we cannot estimate prevalence rates.

\section{Univariate and multivariate analysis}

The distribution of sexual history with male and female partners, sexual practice with women, pregnancy, alcohol 
Table 1 Prevalence of STIs in WSW, stratified by history of sex with men

\begin{tabular}{|c|c|c|c|c|c|c|c|}
\hline \multirow[b]{2}{*}{ STI diagnosed $(n=708)$} & \multirow{2}{*}{$\frac{\text { Cases }}{\text { No }}$} & \multicolumn{2}{|c|}{ Prevalence } & \multicolumn{2}{|c|}{$\begin{array}{l}\text { History of sex with men } \\
(n=581)\end{array}$} & \multicolumn{2}{|c|}{$\begin{array}{l}\text { No history of sex with men } \\
(n=127)\end{array}$} \\
\hline & & $\%$ & $95 \% \mathrm{Cl}$ & No & $\%$ & No & $\%$ \\
\hline Bacterial vaginosis & 222 & 31.4 & (27.9 to 34.8 ) & 183 & (31.5) & 39 & (30.7) \\
\hline Candida species & 130 & 18.4 & (15.5 to 21.2 ) & 109 & (18.8) & 21 & (16.5) \\
\hline Genital warts & 11 & 1.6 & $(0.6$ to 2.5$)$ & 10 & $(1.7)$ & 1 & $(0.8)$ \\
\hline Trichomoniasis & 9 & 1.3 & (0.4 to 2.1$)$ & 8 & $(1.4)$ & 1 & $(0.8)$ \\
\hline Genital herpes & 8 & 1.1 & $(0.3$ to 1.9$)$ & 7 & (1.2) & 1 & $(0.8)$ \\
\hline Chlamydia trachomatis & 4 & 0.6 & $(0.0$ to 1.1$)$ & 4 & $(0.7)$ & 0 & $(0.0)$ \\
\hline Gonorrhoea & 2 & 0.3 & $(-0.1$ to 0.7$)$ & 2 & (0.3) & 0 & $(0.0)$ \\
\hline PID & 2 & 0.3 & $(-0.1$ to 0.7$)$ & 2 & $(0.3)$ & 0 & $(0.0)$ \\
\hline Pediculosis pubis & 0 & - & & - & & - & \\
\hline Any current STI (excluding BV and candida) & 35 & 4.9 & $(3.3$ to 6.5$)$ & 32 & $(5.5)$ & 3 & $(2.4)(p=0.14)$ \\
\hline
\end{tabular}

intake, and smoking is shown in tables on the STI website (www.stijournal/supplemental/ tables A and B). Past or current smoking was the only variable significantly related to diagnosis of any STI at a level of $p<0.05$. Lifetime number of female partners, use of dental dams and employment status were significant at a level of $\mathrm{p}<0.1$ and so were also included in multivariate analysis (table 2). None of the other risk factor variables were significantly associated with diagnosis of any STI.

A non-significant trend was observed with increasing numbers of female sexual partners (the odds ratio for any STI increased by about $40 \%$ for each category increase in numbers of lifetime female partners) (table 2; further tables are available online at http://www.stijournal.com/ supplemental/). Odds of any STI increased with a history of sex with men, although this was not statistically significant. There was a non-significant reduction in the odds of any STI with employment and with the use of dental dams.

\section{DISCUSSION}

\section{Main findings}

Candida and bacterial vaginosis were common. Trichomoniasis, genital warts, and genital herpes were infrequently diagnosed, and pelvic inflammatory disease, chlamydia, and gonorrhoea infections were rare. It appears that trichomoniasis, genital herpes, and genital warts may be sexually transmitted between women, but there is no evidence for woman to woman transmission of gonorrhoea, chlamydia, or PID.

\section{Risk factors}

Heterosexual sexual contact would be expected to increase the prevalence of STI. However, only $6.7 \%$ of WSW had been sexually active with men within the past year. Employment and smoking status are likely to be markers for other factors (for example, choice of higher risk sexual partners). It is plausible that a latex dental dam would reduce the sexual transmission of infection, and that increasing numbers of female partners would increase the risk. These trends need confirming in larger studies.

\section{Limitations of the study}

Incomplete response rates may introduce selection bias. Recall bias and social desirability bias are well recognised in sexual health surveys ${ }^{4}$ and we used convenience sampling, so our results cannot be generalised to all WSW. However, this sample is the largest from the United Kingdom to date and includes a large proportion of asymptomatic women. STI diagnoses were infrequent, so our study lacked the power to analyse all risk factors since some categories contained few subjects.

\section{Comparison with other studies}

No STIs except PID were found in 148 WSW in San Francisco in 1978. ${ }^{5}$ Two much larger studies conducted in Washington State $^{6}$ and Sydney ${ }^{7}$ in the 1990s describe higher prevalence than our results for all STIs except BV and candida. These comparison populations included a high percentage of women who were sex workers ( $14.3 \%$ and $22 \%$, respectively). Only $1.0 \%$ of our sample reported more than 50 lifetime male

Table 2 Crude and adjusted odds ratios for the diagnosis of STIs (excluding BV and candida)

\begin{tabular}{|c|c|c|c|c|c|}
\hline Variable & Cases/total & Crude OR & Adjusted OR & $95 \% \mathrm{Cl}$ & p Value \\
\hline \multicolumn{6}{|c|}{ Number of female sexual partners ever: } \\
\hline $1-5$ & $(10 / 295)$ & 1.0 & 1.0 & & \\
\hline $6-10$ & $(10 / 207)$ & 1.45 & 1.46 & 0.58 to 3.62 & 0.42 \\
\hline$\geqslant 11$ & $(13 / 189)$ & 2.11 & 1.82 & 0.76 to 4.37 & 0.18 \\
\hline \multicolumn{6}{|l|}{ Sex with men } \\
\hline No & $(3 / 127)$ & 1.0 & 1.0 & & \\
\hline Yes & $(32 / 581)$ & 2.41 & 2.00 & 0.60 to 6.79 & 0.26 \\
\hline \multicolumn{6}{|c|}{ Employment status } \\
\hline Unemployed & $(13 / 148)$ & 1.0 & 1.0 & & \\
\hline Employed & $(19 / 457)$ & 0.45 & 0.55 & 0.25 to 1.20 & 0.13 \\
\hline Student & $(1 / 71)$ & 0.15 & 0.18 & 0.02 to 1.47 & 0.11 \\
\hline Other & $(2 / 32)$ & 0.69 & 0.67 & 0.14 to 3.26 & 0.62 \\
\hline \multicolumn{6}{|c|}{ Use of dental dams* } \\
\hline Never & $(33 / 577)$ & 1.0 & 1.0 & & \\
\hline$\geqslant$ Occasionally & $(2 / 120)$ & 0.28 & 0.28 & 0.06 to 1.20 & 0.09 \\
\hline \multicolumn{6}{|c|}{ Past or current smoking } \\
\hline No & $(13 / 424)$ & 1.0 & 1.0 & & \\
\hline Yes & $(22 / 270)$ & 2.8 & 2.49 & 1.18 to 5.26 & 0.02 \\
\hline
\end{tabular}


sexual partners and $1.7 \%$ reported more than 50 lifetime female sexual partners.

Trichomoniasis, genital warts, and genital herpes

Others have described transmission of trichomoniasis, ${ }^{8}$ genital warts, ${ }^{9}$ and genital herpes ${ }^{3}$ between women. Cervical human papillomavirus (HPV) and cervical smear abnormalities have also been noted in women who have no history of sex with men. ${ }^{10}$ Genital herpes, genital warts, and trichomoniasis may have non-sexual modes of transmission. Despite this, sexual transmission between women remains plausible.

\section{Gonorrhoea, chlamydia, and pelvic inflammatory disease}

In common with others ${ }^{3-7}$ we found that infection with gonorrhoea, chlamydia, or PID was present in WSW, but was diagnosed only in those who reported sexual histories with men. These results suggest that routine or repeated screening for gonorrhoea and chlamydia in women who have no history of sex with men may not be necessary.

\section{Comparison with heterosexual clinic attenders}

Studies which included heterosexual female clinic attenders show a lower prevalence of most STIs (except BV) in WSW. ${ }^{3679}$ However, in two of these studies ${ }^{67}$ WSW reported injecting drug use and higher risk partnerships more frequently than heterosexual women, and in the latter the prevalence of hepatitis $\mathrm{B}$ and $\mathrm{C}$ was significantly higher in WSW.

\section{CONCLUSIONS}

Although we have demonstrated a low prevalence of STI in this sample of WSW, lesbians and bisexual women may have sexual histories with men as well as women or histories of injecting drug use, and are therefore vulnerable to sexually transmitted or blood borne infections. These factors outline the importance of taking a thorough history and offering health advice based on behaviour rather than sexual identity. ${ }^{11}$

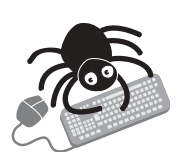

Further tables can be found on the STI website (www.stijournal/supplemental)

Authors' affiliations

J V Bailey, King's College, London, UK

C Farquhar, South Bank University, London, UK

C Owen, Thomas Coran Research Unit, UK

P Mangtani, London School of Hygiene and Tropical Medicine, London, UK

Correspondence to: Dr Julia Vivian Bailey, King's College, London, UK; julia.bailey@kcl.ac.uk

Accepted for publication 11 September 2003

\section{REFERENCES}

1 Ford C, Clarke K. Sexually transmitted infections in women who have sex with women. (Letter) BMJ 1998;316:556-7.

2 Farquhar C, Bailey J, Whittaker D. Are lesbians sexually healthy ? A report of the 'Lesbian Sexual Behaviour and Health Survey'. London, Soc Sci Res Papers No 11, Faculty of Humanities and Social Science, South Bank University, 2001 (www.sbu.ac.uk/fhss/sexuality/reports.shtml).

3 Skinner CJ, Stokes J, Kirlew Y, et al. A case-controlled study of the sexual health needs of lesbians. Genitourin Med 1996:72:277-80.

4 Fenton KA, Johnson AM, McManus S, et al. Measuring sexual behaviour: methodological challenges in survey research. Sex Transm Inf $2001 ; 77: 84-92$.

5 Robertson P, Schachter J. Failure to identify venereal disease in a lesbian population. Sex Transm Dis 1981;8:75-6.

6 Marrazzo JM, Koutsky LA, Handsfield HH. Characteristics of female sexually transmitted disease clinic clients who report same-sex behaviour. Int J STD AIDS $2001 ; 12: 41-6$.

7 Fethers K, Marks C, Mindel A, et al. Sexually transmitted infections and risk behaviours in women who have sex with women. Sex Transm Infect 2000;76:345-9.

8 Kellock DJ, O'Mahony CP. Sexually acquired metronidazole-resistant trichomoniasis in a lesbian couple. Genitourin Med 1996;72:60-1.

9 Edwards A, Thin RN. Sexually transmitted diseases in lesbians. Int J STD AIDS 1990;1:178-81.

10 Marrazzo JM, Koutsky LA, Kiviat NB, et al. Papanicolaou test screening and prevalence of genital human papillomavirus among women who have sex with women. Am J Pub Health 2001;91:947-52.

11 Bailey JV, Farquhar C, Owen C, et al. Sexual behaviour of lesbians and bisexual women. Sex Transm Infect 2003;79:147-50. 